\title{
STOCHASTIC ANALOGUE OF FUNDAMENTAL THEOREM OF SURFACE THEORY FOR SURFACES OF BOUNDED DISTORTION AND POSITIVE CURVATURE
}

\author{
D.S. KLIMENTOV
}

\begin{abstract}
In this paper, we prove a stochastic analogue of Gauss-Peterson-Codazzi equations and provide a stochastic analogue of the fundamental theorem in the theory of surfaces for surfaces of a bounded distortion and a positive curvature. In 1956, I.Ya. Bakelman derived the Gauss-Peterson-Codazzi equations for surfaces of bounded distortion, that is, for the surfaces defined by functions with continuous first derivatives and square summable second generalized derivatives in the sense of Sobolev. In 1988, Yu.E. Borovskii proved that the Gauss-Peterson-Codazzi equations (derived by I.Ya. Bakelman) uniquely determined the surface of a bounded curvature.

The aim of this paper is to present the results of I.Ya. Bakelman and Yu.E. Borovskii in terms of the theory of random processes in the case of a surface of a positive bounded distortion and a positive curvature.

By means of two fundamental forms of the surface, we construct two random processes and derive a system of equations relating the characteristics (transition functions) of these processes. The resulting system is a stochastic analogue of the system of Gauss-PetersonCodazzi equations and is a criterion determining uniquely the surface up to a motion. The generators of random processes are second order operators generated by the fundamental forms of the surface. For instance, if the surface metrics is given by the expression $I=d s^{2}=$ $g_{i j} d x^{i} d x^{j}$, then the generator of the corresponding process is $A=g^{i j} \partial_{i} \partial_{j}$. We establish a relationship between the transition functions of the random process and the generator coefficients. The obtained expressions are substituted into the generalized Gauss-PetersonCodazzi equations, which leads us to the desired result.
\end{abstract}

Keywords: surface of bounded distortion, curvature, random process, transition function of random process, Kolmogorov equation.

Mathematics Subject Classification: 60G99, 53A05

In this paper, we prove a stochastic analogue of Gauss-Peterson-Codazzi equations and provide a stochastic analogue of the fundamental theorem in the theory of surfaces for surfaces of a bounded distortion and a positive curvature. The present work is a continuation of works [1] and [2]. It should be noted that this subject arose as an attempt to construct an analytic geometry on two-dimensional manifolds of a bounded curvature. In what follows we suppose that the curvature of a two-dimensional surface $F$ in a three-dimensional Euclidean space is positive and the surface $F$ is simply-connected and conformally equivalent to a circle. The condition of the a positiveness of the curvature is imposed due to features of constructing diffusion process by a quadratic form. The first and second fundamental forms of the surface $F$

D.S. Klimentov, Stochastic analogue of fundamental theorem of surface theory for surFACES OF BOUNDED DISTORTION AND POSITIVE CURVATURE.

(C)KLimentov D.S. 2019.

The work is supported under a finance support from South Federal University. The author work for South Federal University.

Submitted December 25, 2018. 
are denoted by $I=g_{i j} d x^{i} d x^{j}$ and $I I=b_{i j} d x^{i} d x^{j}$, respectively, where $x^{1}, x^{2}$ are local coordinates on the surface.

The following fundamental theorem of surface theory is well-known [3]:

Gauss-Peterson-Codazzi equations are necessary and sufficient condition for two analytically defined quadratic forms, one being positive definite, to serve as a first and second fundamental forms for some surface, which they determine up to a motion; for its global version we refer to [4].

In 1956, I.Ya. Bakelman in work [5] derived the Gauss-Peterson-Codazzi equations for surfaces of bounded distortion, that is, for the surfaces defined by functions with continuous first derivatives and square summable square generalized second derivatives in the sense of Sobolev. In 1988, Yu.E. Borovsky proved in work [6] that the Gauss-Peterson-Codazzi equations (derived by I.Ya. Bakelman) uniquely determined the surface of a limited curvature.

The paper is organized as follows. In the first section we provide some definitions from the theory of random processes. In the second section part we provide needed information from the theory of two-dimensional manifolds of a bounded curvature (Alexandrov spaces). In the third section we formulate and prove a stochastic analogue of the fundamental theorem of the surface theory for surfaces of a bounded distortion and a positive curvature.

\section{Preliminaries From the theory of RANDOM PROCESSES}

We suppose that the reader is familiar with the definitions of a random, Markov and strictly Markov processes as well as of a diffusion process. We adopt the notations from [7]. For more details on the issues discussed in this section can be found in [7], [8].

We assume that we are given a random space $(\Omega, \mathfrak{F}, P)$.

We consider a manifold (phase space) $(E, \mathfrak{B})$, where $\mathfrak{B}$ is a $\sigma$-field of Borel sets in $E$. A detailed definition of a random process on a manifold can be found in [8].

We give some notations needed in what follows.

Definition 1. [7] A function $P(t, x, \Gamma), t>0, x \in E, \Gamma \in \mathfrak{B}$ is called a transition function if the following conditions are satisfied:

1. For fixed $t$ and $x$, the function $P(t, x, \Gamma)$ is a measure on a $\sigma$-algebra $\mathfrak{B}$.

2. For fixed $t$ and $\Gamma$, the function $P(t, x, \Gamma)$ is a $\mathfrak{B}$-measurable function in the variable $x$.

3. $P(t, x, \Gamma) \leqslant 1$.

4. $P(0, x, E \backslash x)=0$.

5. $P(s+t, x, \Gamma)=\int_{E} P(s, x, d y) P(t, y, \Gamma)$

Let $\mu$ be some measure in the phase space $(E, \mathfrak{B})$.

Definition 2. [7] A function $p(t, x, y), t>0, x, y \in E$, is called a transition density if the following conditions are satisfied:

1. $p(t, x, y) \geqslant 0$.

2. For a fixed $t, p(t, x, y)$ is $\mathfrak{B} \times \mathfrak{B}$-measurable function in the variables $(x, y)$.

3. $\int_{E} p(t, x, y) \mu(d y) \leqslant 1$.

4. $p(s+t, x, z)=\int_{E} p(s, x, y) p(t, y, z) \mu(d y)$.

It is easy to confirm [7] that if $p(t, x, y)$ is a transition density, then the formula

$$
P(t, x, \Gamma)=\int_{\Gamma} p(t, x, y) d y, \quad t>0, \quad P(t, x, \Gamma)=\chi_{\Gamma}, \quad t=0
$$

defines a transition function.

Each transition function is associated with a contracting semigroup $T_{t}$ as follows [7]

$$
T_{t} f(x)=\int_{E} P(t, x, d y) f(y),
$$


where $f \in B, B$ is a set of all bounded measurable functions with natural linear operations and the norm $\|f\|=\sup _{x \in E}|f(x)|$.

Definition 3. [7] An infinitesimal operator of a semigroup $T_{t}$ (of a transition function $P(t, x, \Gamma))$ is the operator $A$ acting according the rule

$$
A f(x)=\lim _{t \rightarrow+0} \frac{T_{t} f(x)-f(x)}{t},
$$

and the domain of the operator $A$ consists of the functions $f$, for which there exists a limit in the right hand side.

If the phase space is equipped with a structure of a smooth manifold, then the infinitesimal operator restricted on twice continuously differentiable functions is called a generator of a random process and in local coordinates $\left(x^{i}\right)$ it reads as

$$
A f(x)=a^{i j} \partial_{i} \partial_{j} f(x)+b^{i} \partial_{i} f(x)-C f(x),
$$

where $\partial_{i}=\frac{\partial}{\partial x^{i}}$, $a^{i j}$ is a semi-definite matrix.

A transition density is related with a generator of a random process by the inverse Kolmogorov equation [7]:

$$
\frac{\partial p}{\partial t}=A p
$$

where the operator $A$ is the generator of a random process introduced above.

It was shown in Chapters 1 and 2 in book [7] that to each Markov process, there are uniquely associated a contracting semigroup, a transition function and an infinitesimal operator.

\section{PRELIMINARIES FROM DIFFERENTIAL GEOMETRY}

A detailed presentation of the theory of two-dimensional manifold with a bounded curvature can be found in book [9]; in this section we follow conventional notations from this book.

Definition 4. [5] A two-dimensional surface $F$ in a three-dimensional Euclidean space is called a smooth surface of a bounded distortion if in a neighbourhood of each its points it can be parametrized as

$$
\vec{r}=\vec{r}\left(x^{1}, x^{2}\right),
$$

where $\vec{r}\left(x^{1}, x^{2}\right)$ is a continuously differentiable vector function of its variables ranging in some domain $D$ in the plane $\left(x^{1}, x^{2}\right)$; this function possesses all its generalized second derivatives locally square summable in $D$ and $\left|\vec{r}_{x^{1}} \times \vec{r}_{x^{2}}\right| \neq 0$ everywhere in $D$. In other words, the function $\vec{r}\left(x^{1}, x^{2}\right)$ and an element of $C^{1} \cap W_{2}^{2}$ inside the mentioned domain.

Let $R$ be a metric space with a metrics $\rho$. If we are given a continuous mapping of a segment $0 \leqslant t \leqslant 1(a \leqslant t \leqslant b)$ into the space $R$, we say that a curve is defined in a parametrization $X(t)$. Different values $t$ can describe the same point $X(t)$. The segment $0 \leqslant t \leqslant 1$ is partitioned into connected components $k_{t}$, each corresponding to the same point $X(t)$. Parametrizations $X(t)$ and $Y(s)$ are called equivalent if there exist a strictly monotone one-to-one correspondence $\phi$ satisfying $X\left(k_{t}\right)=Y\left(\phi\left(k_{t}\right)\right)$.

Definition 5. [9] A curve is a class of equivalent parametrizations.

The length of the curve $X(t), 0 \leqslant t \leqslant 1$ in $R$ can be defined as

$$
\sup \sum_{i=1}^{n} \rho\left(X\left(t_{i-1}\right), X\left(t_{i}\right)\right) \text {, }
$$

where $0=t_{0}<t_{1}<\ldots<t_{n}=1$ is an arbitrary partition of the segment $[0,1]$.

Definition 6. [9] A metrics $\rho$ is called internal if for each two points $X, Y \in R$, the distance $\rho(X, Y)$ is equal to the infimum of the lengths of the curves connecting the points $X, Y$. 
Definition 7. [9] A shortest curve connecting points $X, Y \in R$ is one with the smallest length among all curves with the same ends. A geodesics is a curve being shortest on each sufficiently small part.

Definition 8. [9] A triangle $T=A B C$ in the space $R$ is a set consisting three different points $A, B, C$ called the vertices of the triangle and three shortest curves mutually connecting these points; these curves are the sides of the triangle.

Suppose that in the space $R$, there exists an open in $R$ domain $G$, which is homeomorphic to an open circle in the plane. Let a triangle $T$ lies in this domain and its sides form a simple closed contour, that is, they envelop a domain in $G$. We add this domain to $T$ and we say that $T$ is a triangle homeomorphic to a circle.

Definition 9. [9] We say that a triangle $T$ is boundary convex if any two points of its contour can not be connected by a curve outside $T$ shorter that a piece of the contour connecting these points.

Definition 10. [9] A simple triangle in a domain $G$ is a boundary convex triangle homeomorphic to a circle. Two simple triangles are non-incumbent if they have no common internal points.

Let $L$ and $M$ be two curves in $R$ originating from a same point $O$. Let $X$ and $Y$ be two points moving along $L$ and $M$, respectively. In the plane we construct a triangle $T_{0}$ with sides $\rho(O, X), \rho(O, Y)$ and $\rho(X, Y)$. Such triangle exists since the mentioned distance satisfy the triangle inequality. Let $\gamma(X, Y)$ be an angle opposite to the side $\rho(X, Y)$.

Definition 11. 9] An upper angle (angle) between the curves $L$ and $M$ at the point $O$ is

$$
\overline{\lim }_{X, Y \rightarrow O} \gamma(X, Y) \quad\left(\lim _{X, Y \rightarrow O} \gamma(X, Y)\right) \text {. }
$$

An upper angle in the triangle $T=A B C$ at the vertex $A$ is the upper angle between the shortest curves $A B$ and $A C$.

Definition 12. 9] An upper excess (excess) of a triangle $T$ is the quantity

$$
\bar{\nu}(T)=\bar{\alpha}+\bar{\beta}+\bar{\gamma}-\pi(\nu(T)=\alpha+\beta+\gamma-\pi),
$$

where $\bar{\alpha}, \bar{\beta}, \bar{\gamma}(\alpha, \beta, \gamma)$ are upper angles (angles) in the triangle $T$.

Definition 13. [9] A metric space $R$ is called a two-dimensional manifold of a bounded distortion if the following axioms are satisfied:

1. $R$ is a metric space with an internal metrics;

2. Each point in $R$ has a neighbourhood homeomorphic to a circle in the plane;

3. For each domain $G \subset R$ with a compact closure there exists a number $\nu(G)$ such that for each finite set of mutually non-incumbent simple triangles $T_{i} \subset G$ the inequality holds:

$$
\Sigma_{i}\left|\bar{\nu}\left(T_{i}\right)\right| \leqslant \nu(G)<+\infty .
$$

The following theorem is true.

Theorem 1. [10] A two-dimensional manifold with an internal metrics has a bounded curvature if and only if in each domain $G$ with a compact closure, the induced in each metric $\rho_{G}$ can be uniformly approximated by Riemannian metrics with uniformly bounded absolute curvatures.

A next theorem was proved in [5].

Theorem 2. Each smooth surface of a bounded distortion is a manifold of a bounded curvature in the sense of its internal metrics. 
Now we provide some definitions and results from work [5].

Definition 14. [5] A mean surface $F_{h}$ for a surface $F$ of a bounded distortion with a parametrization $\vec{r}=\vec{r}\left(x^{1}, x^{2}\right)$ is a surface with the parametrization

$$
\vec{r}_{h}\left(x^{1}, x^{2}\right)=\iint_{D-D_{\delta}} \vec{r}(\xi, \eta) \omega_{h}\left(\xi, \eta, x^{1}, x^{2}\right) d \xi d \eta,
$$

where

$$
\begin{aligned}
& \omega_{h}\left(\xi, \eta, x^{1}, x^{2}\right) d \xi d \eta=\left\{\begin{array}{cc}
\frac{1}{H_{h}} e^{\frac{r^{2}}{r^{2}-h^{2}}}, & r<h, \\
0, & r \geqslant h,
\end{array}\right. \\
& H_{h}=\iint_{r \leqslant h} e^{\frac{r^{2}}{r^{2}-h^{2}}} d \xi d \eta, \quad r=\sqrt{\left(x^{1}-\xi\right)^{2}+\left(x^{2}-\eta\right)^{2}} .
\end{aligned}
$$

We introduce the following notations [5]:

$$
\begin{aligned}
& \Gamma_{1}=\frac{1}{2} g_{12} \cdot \frac{\partial g_{22}}{\partial x^{2}}-g_{22}\left(\frac{\partial g_{12}}{\partial x^{2}}-\frac{1}{2} \frac{\partial g_{22}}{\partial x^{1}}\right) ; \\
& \psi\left(x^{1}, x^{2}\right)=\frac{\Gamma_{1}}{\sqrt{g_{11} g_{22}-g_{12}^{2}}} \cdot g_{22},
\end{aligned}
$$

where the subscript $h$ indicates the belonging to the mean surface.

Let $U_{1, \delta}$ be the set of all points in the segment $[a+\delta, b-\delta]$ possessing the following properties: for $u \in U_{1, \delta}$ there exists a subsequence $F_{h_{k}}$ such that

1. For all $c+\delta \leqslant \lambda<\mu \leqslant d-\delta$ the identity holds:

$$
\lim _{h_{k} \rightarrow 0} \int_{\lambda}^{\mu} \psi_{h_{k}}(u, v) d v=\int_{\lambda}^{\mu} \psi(u, v) d v
$$

2. The curve $L_{u}^{1}$ has a finite rotation in the space, which is a function of sets in the curve $L_{u}$ is absolutely continuous;

3. The curves $L_{u, h_{k}}$ converge to $L_{u}$ uniformly with all their tangent lines and have uniformly bounded rotations, which are absolutely equicontinuous.

Theorem 3. [5] On each smooth surface of a bounded distortion in the rectangle $K_{x_{1}^{1} x_{2}^{1}}^{x_{2}^{2} x_{2}^{2}}$ for almost each $x_{1}^{1}, x_{2}^{1} \in U_{1, \delta}$ and for almost each $x_{1}^{2}, x_{2}^{2} \in V_{1, \delta}$ the relations

$$
\begin{aligned}
& \oint_{L} b_{11} d x^{1}+b_{12} d x^{2}=\iint_{\substack{x_{1}^{2} x_{2}^{2} \\
x_{x_{1}^{1} x_{2}^{1}}}} \Gamma_{12}^{1} b_{11}+\Gamma_{12}^{2} b_{12}-\Gamma_{11}^{1} b_{12}-\Gamma_{11}^{2} b_{22} d x^{1} d x^{2}, \\
& \oint_{L} b_{12} d x^{1}+b_{22} d x^{2}=\iint_{\substack{x_{1}^{2} x_{2}^{2} \\
K_{1}^{1} x_{2}^{1}}} \Gamma_{22}^{1} b_{11}+\Gamma_{22}^{2} b_{12}-\Gamma_{21}^{1} b_{12}-\Gamma_{21}^{2} b_{22} d x^{1} d x^{2},
\end{aligned}
$$

hold, where $L$ is the boundary of the rectangle $K_{x_{1}^{1} x_{2}^{1}}^{x_{2}^{2} x_{2}^{2}}, \Gamma_{i j}^{k}$ are generalized second kind Christoffel symbols [5].

Theorem 4. [5] Let $Q$ be some set on a surface of a bounded distortion $F$. The formula

$$
\omega(Q)=\iint_{Q} \frac{b_{11} b_{22}-b_{12}^{2}}{g_{11} g_{22}-g_{12}^{2}} d \sigma,
$$

\footnotetext{
${ }^{1}$ The definition of the rotation of a coordinate line $L_{u}[5]$ is rather cumbersome and we do not provide it here.
} 
holds true, where $\omega(Q)$ is the curvature of $Q, d \sigma$ is the area differential.

In what follows we consider a surface of a bounded distortion and of a positive curvature. The definition of this notion for a two-dimensional manifold of a bounded curvature is rather cumbersome and this is why we do not provide it here. A detailed construction of the notion of the curvature can be found in [9, Ch. 5]. In a smooth case an analogue of the curvature is a Gaussian curvature and its positivity is equivalent to the positive definiteness of the second fundamental form of the surface.

The positivity of the curvature of the surface of a bounded distortion implies that the second fundamental form is positive almost everywhere [5, Sect. 11, 12].

A random process $Y_{t}$ generated by the second fundamental form can be constructed by means of the corresponding Dirichlet form ${ }^{1}[11$. The equivalence of the defining the process by means of the Dirichlet form and by means of the generator was also shown in the same book.

\section{Formulation OF THE MAin RESUlT}

We suppose that on a surface $F$ of a bounded distortion and of a positive curvature uniformly separated from zero, we are given two random processes, $X_{t}$ with a generator $A_{X}=g^{i j} \partial_{i} \partial_{j}$ and $Y_{t}$ with a generator $A_{Y}=b^{i j} \partial_{i} \partial_{j}$. The transition function of the process $X_{t}$ is denoted by $P^{1}(t, x, \Gamma)$, while the same function for the process $Y_{t}$ is denoted by $P^{2}(t, x, \Gamma)$.

Our main theorems are as follows.

Theorem 5. On each smooth surface of a bounded distortion, for almost all $x_{1}^{1}, x_{2}^{1} \in U_{1, \delta}$ and for almost all $x_{1}^{2}, x_{2}^{2} \in V_{1, \delta}$, in the rectangle $K_{x_{1}^{1} x_{2}^{1}}^{x_{2}^{2} x_{2}^{2}}$ the relations hold:

$$
\begin{gathered}
\oint_{L} b_{11} d x^{1}+b_{12} d x^{2}=\iint_{\substack{x_{2}^{2} x_{1}^{2} \\
K_{11}^{1} x_{1}^{2} x_{2}^{1}}} \Gamma_{12}^{1} b_{11}+\Gamma_{12}^{2} b_{12}-\Gamma_{11}^{1} b_{12}-\Gamma_{11}^{2} b_{22} d x^{1} d x^{2}, \\
\oint_{L} b_{12} d x^{1}+b_{22} d x^{2}=\iint_{\substack{x_{1}^{2} x_{2}^{2} \\
K_{x_{1}^{1} x_{2}^{1}}}} \Gamma_{22}^{1} b_{11}+\Gamma_{22}^{2} b_{12}-\Gamma_{21}^{1} b_{12}-\Gamma_{21}^{2} b_{22} d x^{1} d x^{2},
\end{gathered}
$$

where $L$ is the boundary of the rectangle $K_{x_{1}^{1} x_{2}^{1}}^{x_{1}^{2} x^{2}}$, and

$$
\begin{aligned}
& b_{i j}=\Sigma_{k, l=1}^{2} \frac{1}{|I|^{2}} \int P_{t 0}^{1}(t, x, d y) \frac{y_{i} y_{k}}{1+\delta_{i k}} \int P_{t 0}^{1}(t, x, d y) \frac{y_{j} y_{l}}{1+\delta_{j l}} \int P_{t 0}^{2}(t, x, d y) \frac{y_{k} y_{l}}{1+\delta_{k l}}, \\
& \Gamma_{i j, k}=\frac{1}{2}\left(\frac{\partial g_{i k}}{\partial x^{j}}+\frac{\partial g_{j k}}{\partial x^{i}}-\frac{\partial g_{i j}}{\partial x^{k}}\right), \quad \Gamma_{i j}^{l}=g^{k l} \Gamma_{i j, k}, \\
& |I|=\int P_{t 0}^{1}(t, x, d y) \frac{y_{1}^{2}}{2} \cdot \int P_{t 0}^{1}(t, x, d y) \frac{y_{2}^{2}}{2}-\left[\int P_{t 0}^{1}(t, x, d y) y_{1} y_{2}\right]^{2},
\end{aligned}
$$

the derivatives are understood in the sense of Sobolev, the subscript t0 denotes the derivative in $t$ at $t=0$, and

$$
g_{i j}=\frac{1}{|I|} \int P_{t 0}^{1}(t, x, d y) \frac{y_{i} y_{j}}{1+\delta_{i j}} .
$$

Theorem 6. Let $Q$ be some set on a surface $F$ of a bounded distortion. The formula holds:

$$
\omega(Q)=\iint_{Q} \frac{b_{11} b_{22}-b_{12}^{2}}{g_{11} g_{22}-g_{12}^{2}} d \sigma
$$

\footnotetext{
${ }^{1}$ The construction of the random process by means of the Dirichlet form is rather cumbersome [11, Ch. 1] and we do not provide it here.
} 
where $\omega(Q)$ is the curvature on $Q, d \sigma$ is the differential of the area,

$$
\begin{aligned}
b_{i j} & =\Sigma_{k, l=1}^{2} \frac{1}{|I|^{2}} \int P_{t 0}^{1}(t, x, d y) \frac{y_{i} y_{k}}{1+\delta_{i k}} \int P_{t 0}^{1}(t, x, d y) \frac{y_{j} y_{l}}{1+\delta_{j l}} \int P_{t 0}^{2}(t, x, d y) \frac{y_{k} y_{l}}{1+\delta_{k l}}, \\
g_{i j} & =\frac{1}{|I|} \int P_{t 0}^{1}(t, x, d y) \frac{y_{i} y_{j}}{1+\delta_{i j}}, \\
|I| & =\int P_{t 0}^{1}(t, x, d y) \frac{y_{1}^{2}}{2} \cdot \int P_{t 0}^{1}(t, x, d y) \frac{y_{2}^{2}}{2}-\left[\int P_{t 0}^{1}(t, x, d y) y_{1} y_{2}\right]^{2} .
\end{aligned}
$$

Theorem 7. Two random processes $X_{t}$ and $Y_{t}$ determine uniquely a surface of a bounded distortion (up to a position in the space) if and only if equations (3), (4) hold true.

\section{PROOF OF MAIN RESUlT}

The proofs of the theorems are based on several lemmata.

Lemma 1. Contravariant coefficients of the first fundamental form are related with the transition functions of the process $X_{t}$ by the following formulae:

$$
\begin{aligned}
g^{11} & =\int \frac{d}{d t}\left(P^{1}(t, x, d y)\right)_{t=0} \frac{y_{1}^{2}}{2}, \\
g^{12} & =\int \frac{d}{d t}\left(P^{1}(t, x, d y)\right)_{t=0} y_{1} y_{2}, \\
g^{22} & =\int \frac{d}{d t}\left(P^{1}(t, x, d y)\right)_{t=0} \frac{y_{2}^{2}}{2} .
\end{aligned}
$$

Proof. In book [7], the formula $T_{t} f=\int P(t, \vec{x}, d y) f(y)$ was established. By definition, the infinitesimal diffusion operator $X_{t}$ is defined by identity

$$
A_{X} f=\lim _{t \rightarrow+0} \frac{T_{t} f-f}{t} .
$$

Let us consider this identity in more details. We fix a function $f$ in the domain of the generator. For twice continuously differentiable functions $f$, the left hand side is well-defined and is continuous, therefore, the limit in the right hand side exists and is equal to

$$
A_{X} f=\frac{d}{d t}\left[T_{t} f\right]_{t=0}
$$

Here we have employed the identity $T_{0} f=f$. In our case, the generator of the process $X_{t}$ reads as $A_{X} f=g^{i j} \partial_{i} \partial_{j} f$. Choosing the function $f$ as $f=\frac{y^{i} y^{j}}{1+\delta^{i j}}$ and substituting it into the latter identity for the generator, we obtain the statement of the lemma:

$$
g^{i j} \partial_{i} \partial_{j} f(x)=\int \frac{d}{d t}\left(P^{1}(t, x, d y)\right)_{t=0} f(y) .
$$

We observe that we interchange the derivation and the integral by the Lebesgue theorem on a majorized convergence [12].

In what follows, in order not to complicate the notations, the derivation in time at $t=0$ is denoted by the subscript $t 0$. 
Lemma 2. Covariant coefficients of the first fundamental form of the surface read as

$$
\begin{aligned}
& g_{11}=\frac{1}{|I|} \int P_{t 0}^{1}(t, x, d y) \frac{y_{2}^{2}}{2}, \\
& g_{22}=\frac{1}{|I|} \int P_{t 0}^{1}(t, x, d y) \frac{y_{1}^{2}}{2}, \\
& g_{12}=-\frac{1}{|I|} \int P_{t 0}^{1}(t, x, d y) y_{1} y_{2},
\end{aligned}
$$

where

$$
|I|=\int P_{t 0}^{1}(t, x, d y) \frac{y_{1}^{2}}{2} \cdot \int P_{t 0}^{1}(t, x, d y) \frac{y_{2}^{2}}{2}-\left[\int P_{t 0}^{1}(t, x, d y) y_{1} y_{2}\right]^{2} .
$$

Proof. The statement is immediately implied by the relation

$$
g_{i l} g^{l j}=\delta_{i}^{j},
$$

where $\delta_{i}^{j}$ is the Kronecker delta.

Lemma 3. Contravariant coefficients of the second fundamental form of the surface are related with transitions functions of the process $Y_{t}$ by the relations

$$
\begin{aligned}
b^{11} & =\int P_{t 0}^{2}(t, x, d y) \frac{y_{1}^{2}}{2}, \\
b^{12} & =\int P_{t 0}^{2}(t, x, d y) y_{1} y_{2}, \\
b^{22} & =\int P_{t 0}^{2}(t, x, d y) \frac{y_{2}^{2}}{2} .
\end{aligned}
$$

Proof. The proof reproduces that of Lemma 1 literally with the only exception: the values of the generator $A_{Y}$ on twice differentiable functions are generally speaking square summable but this makes no influence of the further arguing.

Lemma 4. Contravariant coefficients of the second fundamental form of the surface read as

$$
b_{i j}=\Sigma_{k, l=1}^{2} \frac{1}{|I|^{2}} \int P_{t 0}^{1}(t, x, d y) \frac{y_{i} y_{k}}{1+\delta_{i k}} \int P_{t 0}^{1}(t, x, d y) \frac{y_{j} y_{l}}{1+\delta_{j l}} \int P_{t 0}^{2}(t, x, d y) \frac{y_{k} y_{l}}{1+\delta_{k l}} .
$$

Proof. The proof is immediately implied by a known formula $b_{i j}=g_{i k} g_{j l} b^{k l}$ and Lemmata 1 3.

Lemma 5. The Christoffel coefficients are calculated by the formulae

$$
\Gamma_{i j, k}=\frac{1}{2}\left(\frac{\partial g_{i k}}{\partial x^{j}}+\frac{\partial g_{j k}}{\partial x^{i}}-\frac{\partial g_{i j}}{\partial x^{k}}\right), \quad \Gamma_{i j}^{l}=g^{k l} \Gamma_{i j, k},
$$

where the derivatives are treated in the Sobolev sense, and

$$
g_{i j}=\frac{1}{|I|} \int P_{t 0}^{1}(t, x, d y) \frac{y_{i} y_{j}}{1+\delta_{i j}} .
$$

Proof. In work [5], the existence of the Christoffel symbols was proved for a surface of a bounded distortion and it was shown that they were calculated by the above formulae. Then the statement of the lemma follows easily Lemmata 1, 2.

The proofs of the main theorem obviously follow the mentioned lemmata and the results of works [5], 6]. 


\section{BIBLIOGRAPHY}

1. D.S. Klimentov. Stochastic analogue of fundamental theorem of surface theory for surfaces of positive curvature // Izvestia VUZov. Severo-Kavkazskii region. Estest. nauki. 6, 24-27 (2013). (in Russian).

2. D.S. Klimentov. Stochastic analogue of fundamental theorem of surface theory for surfaces of non-zero mean curvature // Izvestia VUZov. Severo-Kavkazskii region. Estest. nauki. 1, 15-18 (2014). (in Russian).

3. P.K. Rashevsky. Course of differential geometry. GONTI, Moscow (1939). (in Russian).

4. S. Sasaki. A global formulation of the foundamental theorem of the theory of surfaces in three dimensional Euclidean space // Nagoya Math J. 13, 69-82 (1958).

5. I.Ya. Bakelman. Differential geometry of smooth non-regular surfaces // Uspekhi Matem. Nauk. 11:2(68), 67-124 (1956). (in Russian).

6. Yu.E. Borovskii. Pfaff systems with the coefficients in $L_{n}$ and their geometric applications // Sibir. Matem. Zhurn. 24:2, 10-16 (1988). (in Russian).

7. E.B. Dynkin. Theory of Markov processes. Fizmatlit, Moscow (1963). [Dover Publications, Mineola, New York (2006).]

8. N. Ikeda, S. Watanabe. Stochastic differential equations and diffusion processes. North-Holland Publ. Co., Amsterdam (1981).

9. A.D. Aleksandrov, V.A. Zalgaller. Two-dimensional manifolds of bounded curvature (Foundations of the intrinsic geometry of surfaces). Trudy Mat. Inst. Steklov. 63, 3-262 (1962). (in Russian).

10. Yu.G. Reshetnyak. Two-dimensional manifolds of bounded curvature // Itogi Nauki Tekhniki. Ser. Sovrem. Probl. Mat. Fund. Napr. 70, 7-189 (1989). (in Russian).

11. M. Fukushima, Y. Oshima, M. Takeda. Dirishlet forms and symmetric Markov processes. Walter de Gruyter, Berlin (1994).

12. A.N. Kolmogorov, S.V. Fomin. Introductory real analysis. Nauka, Moscow (1976). [Prentice-Hall, Inc., Englewood Cliffs, New-Jersey (1970).]

Dmitrii Sergeevich Klimentov

Institute of Mathematics, Mechanics and Computer Sciences,

South Federal University,

Milchakova str. 8a,

344000, Rostov-on-Don, Russia

E-mail: dklimentov75@gmail.com 